Meta

Journal des traducteurs

Translators' Journal

\title{
Structures lexicales, terminologies techniques et glossaires contextuels multilingues
}

\section{Roger Goffin}

Volume 18, numéro 1-2, mars 1973

Actes du deuxième colloque international de linguistique et de traduction. Montréal, 4-7 octobre 1972

URI : https://id.erudit.org/iderudit/002931ar

DOI : https://doi.org/10.7202/002931ar

Aller au sommaire du numéro

\section{Éditeur(s)}

Les Presses de l'Université de Montréal

\section{ISSN}

0026-0452 (imprimé)

1492-1421 (numérique)

Découvrir la revue

\section{Citer cet article}

Goffin, R. (1973). Structures lexicales, terminologies techniques et glossaires contextuels multilingues. Meta, 18(1-2), 237-253.

https://doi.org/10.7202/002931ar d'utilisation que vous pouvez consulter en ligne. 


\section{Structures lexicales, terminologies techniques et glossaires contextuels multilingues}

Dans un article riche en réflexions suggestives qu'il consacra à une analyse systématique des problèmes concernant la structure lexicale ${ }^{1}$, Eugenio Coseriu a énoncé à propos des «terminologies » un certain nombre d'hypothèses de travail, toujours extrêmement fructueuses mais parfois quelque peu fragiles, qui constitueront le point de départ et la clé des analyses que nous tenterons dans le présent exposé.

Ces hypothèses ont pour effet de rejeter l'étude des terminologies dans le domaine de la linguistique dite externe ou du moins de les isoler de l'ensemble du lexique. Par voie de conséquence, la lexicologie structurale risque de négliger, dans un premier temps, les résultats des études consacrées aux terminologies, même si elle se réserve le droit, comme le dit Coseriu lui-même, d'y revenir dans une étape ultérieure ${ }^{2}$.

Or, il n'est pas inutile de supposer que la masse des unités lexicales se répartit en un certain nombre de grands ensembles de vocabulaires, au sein desquels s'organisent des sous-ensembles plus ou moins cohérents et il n'est pas absurde d'admettre que les terminologies techniques, chacune couvrant un domaine de l'activité humaine, constituent un secteur privilégié d'études lexicologiques. Elles représentent du moins une première tentative de structuration du lexique général dont la linguistique pourrait sans doute faire son profit.

Le structuralisme est à la mode. On le retrouve partout : linguistique structurale de F. de Saussure, histoire structurale de Dumézil, psychanalyse structurale de J. Lacan, anthropologie structurale de C. Lévi-Strauss. Au fond, on cherche à voir clair, à ordonner et à classer la masse des données, et notamment, celles du lexique. Encouragés par les succès parfois éclatants de la méthode structuraliste dans la description phonologique et dans la description grammaticale, les linguistes souhaiteraient aboutir à une ordonnance du lexique. Ils s'achoppent à l'analyse structurale du lexique où les résultats ont été moins probants et les orientations assez hétérogènes ${ }^{3}$. Beaucoup de linguistes vont d'ailleurs jusqu'à nier, en raison

1. E. Coseriu, « Structure lexicale et enseignement du vocabulaire », les Théories linguistiques et leurs applications, Strasbourg, Conseil de la coopération culturelle, AIDELA, 1967, p. $9-51$.

2. Ibid., p. 18 .

3. Voir notamment A. Rey, la Lexicologie, Paris, Klincksieck, "Lectures ", 1970, le chapitre : "Visions du lexique $»$, p. 69-75; A. Martinet, la Linguistique, guide alphabétique, Paris, Denoël, 1969, le chapitre de P. Pupier : "Le lexique », p. 88-201; J. Rey-Debove, 
du caractère non fini des unités lexicales, l'existence de toute structure systématique du lexique. Pour L. Bloomfield notamment, le lexique représente, en face du système de la grammaire, l'irrégularité et l'hétérogénéité : «le lexique est en réalité... une liste d'irrégularités fondamentales ${ }^{4}$ ». Pour L. Hjelmslev « le vocabulaire reste capricieux et juste le contraire d'une structure ${ }^{5} \gg$. A. Martinet abonde dans le même sens : "le lexique proprement dit semble beaucoup moins facile à réduire à une structuration, dès qu'on a épuisé certains domaines particulièrement favorables tels que les termes de parenté, les numéraux et quelques autres ${ }^{6} \gg$. On s'est demandé quelles sont les raisons de cette faillite, d'autant que le mot lexématique, c'est-à-dire le lexème porteur de la fonction lexicale est plus concrètement présent que le phonème et qu'il faut compter avec la longue pratique des lexicographes dont les descriptions obéissaient, il est vrai, à des règles conventionnelles non scientifiques. La difficulté réside tout d'abord dans le nombre considérable d'unités lexicales à traiter (le dictionnaire Webster contient plus de 600000 mots, à l'inverse du nombre plus restreint de paradigmes grammaticaux et du nombre de phonèmes qui, en anglais, ne dépasseraient pas 44 ou 45), ensuite dans leur organisation complexe (on constate que les relations lexicales sont extrêmement complexes, souvent imprécises, parfois inextricables), enfin dans le caractère subjectif d'un système éventuel, si bien qu'il faudrait parler d'une somme de systèmes individuels. En effet, les signifiés ne se rangent pas suivant un système identique chez tous les locuteurs. E. Buyssens a mis en évidence l'importance du facteur individuel dans le sous-système qu'est le vocabulaire ?

E. Coseriu a inséré ses considérations sur les terminologies dans un paragraphe intitulé "Choses et langages ${ }^{8}$ ». Ce n'est pas le fait du hasard, car il met ainsi d'entrée de jeu le linguiste en garde ; il faudra distinguer dans ce que l'on a tendance à appeler signification, entre ce qui est dû à la connaissance des choses en tant que telles et ce qui est dû au langage et il faudra déterminer quelles sont les structurations du signifié et quelles sont les associations sémantiques qui sont dues à des analyses non linguistiques des objets et à des états de choses réels. L'alternative est la suivante : ou bien la connaissance est en accord avec la langue et s'intègre à elle, comme le croit le structuraliste, qui s'interdit de faire appel à la connaissance; ou bien la langue et la connaissance ont leurs systèmes indépendants et il faut faire appel à la connaissance de la réalité extra-linguistique pour expliquer les faits linguistiques. Comme l'a très bien montré E. Buyssens, le système des signes linguistiques exprimant la connaissance ne s'accorde pas toujours au système des idées dans lequel s'organise la connaissance. Rappelons l'exemple

«Le domaine du dictionnaire », la Lexicographie, Langages 19, septembre 1970, p. 3-34; C. Ducháček, "Quelques observations sur la structure du lexique », Mélanges G. Straka, Strasbourg, 1970 , p. $200-210$; J. Dubois, "Les problèmes du vocabulaire technique ", Cahiers de lexicologie, vol. II, $\mathrm{n}^{\circ} 9,1966$.

4. L. Bloomfield, le Langage (Language), trad. A. Rey, cité d'après A. Rey, op. cit., p. 75.

5. L. Hjelmslev, Pour une sémantique structurale, travaux du Cercle linguistique de Copenhague, 1957, vol. XII, p. 97, cité d'après A. Rey, op. cit., p. 76.

6. A. Martinet, «Structural Linguistics», Anthropology today, Chicago, Hocker, 1953, cité d'après A. Rey, op. cit., p. 129.

7. E. Buyssens, « Le structuralisme et l'arbitraire du signe», Studii si cercetari Linguistice, Omagiu lui HL. Graur, Bucarest, Academia republicii populare romîne, 1960, 3, anul. XI, p. $403-416$.

8. E. Coseriu, «Structure lexicale... », p. 22. 
de Buyssens qui compare les groupes pomme de terre et motte de terre, l'un correspondant à un élément unique de connaissance, l'autre à un complexe d'éléments de connaissance ${ }^{9}$. On peut conclure cette introduction par la phrase de Coseriu : «la langue commune classe, ordonne la réalité suivant les critères qui lui. sont propres et le fait selon des intérêts et des attitudes humaines ${ }^{10} \gg$. Corollairement, il faudra faire la distinction entre les rapports de signification (Bedeutung) (qui ne sont que les rapports entre les signifiés des signes linguistiques), depuis de Saussure on parle alors de «valeur», et les rapports de désignation (Bezeichnung) qui sont les rapports entre les signes linguistiques et les objets, c'est-à-dire la réalité. C'est ce que Buyssens appelle le « désignant», ou ce qui, dans le signifié, assure le lien avec la connaissance de la réalité ${ }^{11}$.

À la lumière de ces remarques liminaires, nous souhaiterions examiner un peu plus en détail quatre hypothèses émises par E. Coseriu à propos des terminologies.

Première hypothèse : "les terminologies scientifiques n'appartiennent pas au langage ni, par conséquent, aux structurations lexicales au même titre que les mots usuels \$ (p. 15).

Il est généralement admis que le terme technique se distingue du mot de la langue commune par son caractère monosémique, c'est-à-dire par sa référence univoque au signifié, par sa spécificité dans un groupe de locuteurs et par l'absence de toute qualité esthétique ${ }^{12}$. Le terme scientifique a un «désignant» bien précis, du moins on le suppose généralement, et une valeur souvent négligeable.

Le terme uranium désigne un élément de connaissance qui suffit à déterminer son signifié. Ceci vaut pour presque tous les éléments de la classification de Mendeleev quoiqu'il y ait aujourd'hui dispute autour de l'élément 102, baptisé nobélium. Les Suédois disent l'avoir découvert en 1957, les Américains en 1958, les Russes quelques années plus tard et prétendent que les laboratoires soviétiques sont les seuls à avoir effectué les expériences convaincantes. Ils suggèrent pour leur part de rebaptiser le nobélium et de lui donner, sans esprit chauvin, le nom de joliotium. La relation simple : un signifiant - un signifié, si typique pour le terme technique, ne vaut pas pour tous ces termes d'application plus générale qui entrent dans le vocabulaire de plusieurs sciences et techniques et dont l'imprécision sémantique ne peut être levée que par le contexte extra-linguistique. Cette prétendue monosémie est donc plutôt une polyvalence. A. Phal a montré que

9. E. Buyssens, "Le structuralisme... », p. 406.

10. E. Coseriu, "Structure lexicale... », p. 22.

11. E. Buyssens, "Le structuralisme... », p. 406.

12. Nous renvoyons notamment aux études suivantes : L. Guilbert, la Formation du vocabulaire de l'aviation, Paris, Larousse, 1965 , p. 335 ss.; E. Benes, « Syntaktische Besonderheiten der deutschen wissenschaftlichen Fachsprache, Probleme der Sprachwissenschaft, La Haye et Paris, Mouton, 1971, p. 461-475; W. Reinhardt, " Eigentümlichkeiten der Fachsprachen ", ibid., p. 452-460; V. Petioky, "Einige Gedanken zum Thema Fachsprachen ", Begegnung zwischen Praxis und Lehre. Die Ausbildung zum Ubersetzer und Dolmetscher, Wiesbaden, Brandstetter, 1970, p. 55-73; A. Phal, "Le vocabulaire général d'orientation scientifique : essai de définition et méthode d'enquête ", les Langues de spécialité, analyse linguistique et recherche pédagogique, Strasbourg, AIDELA, 1970, p. 94-107. 
l'existence d'éléments sémiques fondamentaux expliquait pourquoi les termes se rencontrent dans plusieurs langues spéciales ${ }^{13}$. De plus, la relation : un signifiant - un signifié ne se retrouve pas dans la période initiale de génèse d'une réalité scientifique nouvelle. Une terminologie en gestation, comme celle de l'atome ou de l'information, se caractérise davantage par un foisonnement de lexèmes synonymes qui font que la «valeur $\gg$ du terme, au sens saussurien, joue un rôle de première importance.

C'est ainsi que facteur de désavantage, facteur de flux neutronique et rapport de désavantage sont utilisés pour désigner le rapport entre les densités de flux neutronique moyennes dans un matériau déterminé et dans le combustible. État normal et état fondamental désignent l'état d'un système dont l'énergie est la plus basse possible. Enfin, paramètre de ralentissement est synonyme du calque anglais décrément logarithmique moyen de l'énergie ${ }^{14}$.

C'est pour une très grande part à cause de la diffusion de l'information scientifique que la langue technique tend de plus en plus à sortir d'un milieu circonscrit qui était le sien et à bousculer l'ensemble du vocabulaire. Cette langue a tendance à se séculariser ${ }^{15}$. Les mutations $\mathrm{du}$ monde ont eu pour effet de libérer la langue scientifique de tout solipsisme exagéré. Nous avons montré ailleurs le caractère ambivalent de ce langage, tout à la fois ésotérique par suite du caractère cryptique de la recherche et exotérique par suite de sa diffusion élargie et de son caractère pragmatique.

Il existe entre la terminologie scientifique et la langue commune une interaction profitable aux deux parties ${ }^{16}$. La langue commune accueille très volontiers de nouveaux mots techniques, qui sont de ce fait susceptibles d'être structurés de la même manière que les mots usuels. Le jargon des sciences ombrage la langue contemporaine et à peine les techniciens ont-ils accordé l'investiture à certains termes techniques que la presse s'en empare pour les faire tomber, par un emploi métaphorique, dans le domaine public. En revanche, les terminologies scientifiques ne sont pas coupées du lexique général dans lequel elles puisent assez largement. Le mot en devenant terme subit une inflexion sémantique à la fois du fait qu'il se trouve inséré dans un contexte spécialisé qui lui donne une empreinte propre et du fait qu'il est, dans son environnement lexical immédiat, accompagné d'éléments que l'on n'a pas l'habitude de retrouver en sa compagnie dans la langue commune. Lorsqu'il immigre dans la terminologie spéciale, le mot prend la teinte de son milieu d'adoption mais conserve, le plus souvent, les principaux facteurs sémiques de son origine; il reste donc lié, du moins par le noyau sémique fondamental, à la langue commune.

13. A. Phal, « Recherche au CREDIF : la part du lexique commun dans les vocabulaires scientifiques et techniques $"$, Langue française : le lexique, no 2, 1969, p. 74-77.

14. R. Goffin, "Réflexions sur la terminologie de l'atome ", Equivalences, Bruxelles, 1, 1970, p. 15-27.

15. R. Goffin, a La terminologie multilingue et la syntagmatique comparée au service de la traduction technique $\gg$, Linguistica Antverpiensia, $\mathrm{n}^{\circ} 2,1968$, p. 189-205.

16. B. E. Vidos, "La place du vocabulaire technique dans le thesaurus de la langue française ", Lexicologie et lexicographie françaises et romanes (Actes du Colloque de Strasbourg, 1957), Paris, 1961, p. 185-194. 
Les atomistes ont donné un sens nouveau à nombre de mots de la tribu. Ils utilisent l'adjectif chaud (E. hot, D. heiss), dans atome chaud, laboratoire chaud, pour qualifier un degré de radioactivité élevé. Ce néologisme sémantique conserve, en dépit de son usage insolite, un sème très proche du sème fondamental d'origine. En technologie des réacteurs, l'adjectif dur (E. hard, D. hart) est utilisé pour qualifier un rayonnement de grande énergie; l'adjectif mou (E. soft, D. weich) pour qualifier un rayonnement de faible énergie. Une substance opaque (E. opaque, D. undurchlässig) est une substance qui s'oppose au passage d'un rayonnement. Le qualificatif antonymique est transparent (E. transparent, D. durchlässig). Le physicien dira donc : le bismuth est opaque aux rayons gamma mais transparent aux neutrons. En technologie des réacteurs, le corps noir (E. black, D. schwarz) est le corps qui absorbe pratiquement tous les neutrons incidents d'énergie spécifiée. La locution "être noir aux neutrons thermiques " (E. to be black to thermal neutrons) signifie donc que ces neutrons sont absorbés. Si une partie des neutrons n'est pas absorbée, le corps est dit gris (E. grey body).

L'âge des neutrons (E. age, D. Alter) est la valeur de l'aire de ralentissement d'un neutron, calculée par la théorie de l'âge de Fermi. La lacune (E. vacancy, D. Leerstelle) est un défaut ponctuel se produisant quand un emplacement normalement occupé par un atome se trouve inoccupé.

Le pelage (E. chemical jacket removal; D. chemisches Enthülsen) est l'opération consistant à séparer le combustible de sa gaine par attaque chimique de celle-ci. Le gavage du réacteur est la technique qui consiste à introduire dans un réacteur une quantité supplémentaire de combustible de façon que la proportion entre le combustible et le modérateur soit supérieure à la valeur optimale.

Comme l'écrit justement $\mathrm{A}$. Phal «ces termes conservent intact leur noyau sémique en dépit des phénomènes de réfringence qui les affectent quand ils sont transposés dans tel ou tel contexte particulier ${ }^{17}$ ». Ils sont donc à coup sûr justiciables d'une structuration sémantique. Certains mots du vocabulaire courant acquièrent leur spécificité du fait qu'ils sont associés à d'autres mots, souvent de nature adjectivale, en compagnie desquels on ne les retrouve pas dans la langue générale. C'est la dérivation syntagmatique qui se caractérise par une forte cohérence interne. Le poison nucléaire (E. nuclear poison, D. Neutrongift) est une substance qui, par suite de sa section efficace d'absorption des neutrons, réduit la réactivité d'un réacteur. Les atomistes ont forgé le poison consommable, introduit à dessein dans un réacteur pour contribuer au contrôle des variations. Dans les grandes familles de nucléides, il ne convient pas toujours d'appeler les ascendants par leur nom : l'expression père nucléaire, qui désigne le nucléide radioactif considéré par rapport aux produits de sa désintégration, devient en allemand Mutternuklid et sans sexe précis nuclear parent en anglais. Le cimetière fertile ou radioactif est un emplacement aménagé pour stocker des objets radioactifs inutilisables (E. radioactive burial ground).

Il est aussi intéressant de constater que l'interdépendance existant entre la terminologie scientifique et le stock lexical commun varie sensiblement d'une langue

17. A. Phal, " Recherche au CREDIF... ", p. 75. 
à l'autre. La position qu'occupe un vocabulaire technique au sein du lexique commun ne semble pas être la même dans toutes les langues. Le français technique s'est détaché du lexique commun d'une manière moins radicale que ne l'a fait le russe technique. Comme l'a montré V. G. Gak ${ }^{18}$, cette langue tend, à tout prix, à faire le départ entre le mot de la langue commune et le mot de la langue spéciale par l'emploi d'une marque formelle, notamment la forme diminutive, chaque fois qu'il s'agit de dénommer métaphoriquement les concepts techniques. Ainsi, le mot désignant la manivelle dérive du mot ruka (bras) et prend la forme du diminutif ručka. S'ejka diminutif de šeja (= cou) signifie "goulot » et rožok diminutif de rog (corne) signifie « bec de gaz », «cornet acoustique ». Si la forme diminutive est celle qui est utilisée dans la langue commune, par exemple butylka (bouteille), la langue technique utilisera la forme pleine butyl (au sens de bouteille, bonbonne) ${ }^{19}$.

Quant aux raisons qui nous portent à mettre en évidence le danger de séparer d'une manière trop radicale les terminologies de la structure d'ensemble du lexique général et à admettre l'existence de larges zones d'interférence, nous en distinguerions trois : 1) le stock lexical spécial se trouve étroitement lié au vocabulaire commun, les liens de filiation étant différents d'une langue à l'autre ; 2) le même terme appartient très souvent à plusieurs langues spéciales différentes, et, comme l'a montré J. C. Gardin, tout terme technique appartient à une organisation sémantique multivoque ${ }^{20}$; 3) si le lexicographe repousse, parfois à juste titre, dans des glossaires spéciaux tous les termes appartenant aux connaissances spéciales, il aura intérêt dans ses investigations sur la structuration du lexique à ne pas traiter ces termes en parias qui vivent en marge du lexique.

Deuxième hypothèse : «les terminologies ne sont aucunement «structurées 》, elles sont de simples nomenclatures énumératives correspondant à des délimitations dans les objets $\gg$ (p. 15).

En assimilant les terminologies scientifiques à de simples nomenclatures énumératives, telles que les classifications de la botanique ou de la zoologie, la nomenclature chimique ou encore la taxinomie biologique, le linguiste met sur un pied d'égalité deux classifications de nature différente. Toutes les terminologies ne représentent pas, comme celles de la chimie et de la botanique, des ensembles délibérément organisés dans lesquels chaque terme symbolise en quelque sorte sa nature, ses caractéristiques et la place qu'il occupe dans la nomenclature extralinguistique. Il est vrai que le mot terminologie a souvent été utilisé dans des acceptions assez divergentes, il est vrai que le Petit Larousse confond les mots terminologie et nomenclature, qu'il assortit d'une définition quasi identique : La nomenclature y est définie comme «l'ensemble des termes techniques d'une science ou d'un art», la terminologie comme «l'ensemble des termes particuliers à une science ou un art ${ }^{21} ! »$.

18. V. G. Gak, Besedy o francuzskom slove, Mezdunarodny odnochenija, Moscou, 1967, cité dans la traduction de Jean Charade, Réflexions sur le mot français, mémoire de licence à l'I.S.T.I., Bruxelles, 1970.

19. J. Charade, op. cit., p. 99-100.

20. J. C. Gardin, le Fichier mécanographique de l'outillage, Beyrouth, 1956. Voir à ce propos : G. Mounin, les Problèmes théoriques de la traduction, Paris, 1963, p. 112-124.

21. Nous empruntons cette remarque à J. Maillot, la Traduction scientifique et technique, Paris, Eyrolles, 1970 , p. 143 ss. 
La notion de terminologie a fait l'objet d'une recommandation de l'organisation internationale de normalisation, plus spécialement élaborée par son comité technique ISO/TC 37 «terminologie 22 ». Les auteurs de la norme ont distingué deux sens différents et les ont répartis sur deux rubriques (37 et 38 ). Premièrement, la terminologie comme science est le «domaine du savoir consacré à la formation et à la dénomination des notions, soit dans un domaine spécial, soit pour tous les domaines $»$. Deuxièmement, la terminologie est définie comme «l'ensemble de termes représentant un ensemble de notions liées, surtout dans un domaine spécial ». La première définition s'applique à caractériser un domaine spécialisé de l'activité de l'esprit humain ayant trait à la formation et à la dénomination des notions, ainsi qu'à la création de termes nouveaux (la néologie). En fait, c'est la science qui étudie les relations entre signifiants et signifiés. On sait que ces liens sont complexes. Ce sens ne nous retiendra pas ici.

Dans sa seconde acception, la terminologie est un ensemble de mots techniques ou termes appartenant à une langue spéciale qui n'est pas d'un usage courant dans la langue commune. Pour les auteurs de la recommandation, le terme nomenclature est employé dans certains domaines, en biologie par exemple, dans un sens similaire mais non identique à celui de terminologie. La nomenclature se « rapporte aux noms de plantes et des animaux, tandis que la terminologie se rapporte à leurs parties et caractéristiques ».

J. Maillot pour sa part définit la nomenclature comme \&les moyens et méthodes permettant de distinguer les uns des autres les objets faisant partie d'un même ensemble, en général à l'aide de procédés graphiques », alors que la terminologie est la «science qui s'attache avant tout à identifier un terme en décrivant la notion qu'il représente, sans recourir à des moyens accessoires, tels qu'une illustration, un synonyme, son équivalent dans une autre langue ${ }^{23} \gg$. Les catalogues illustrés et les répertoires de matériels dont l'agent principal est la représentation visuelle sont de bons exemples de ce que l'on peut entendre par ouvrage de nomenclature.

Une autre distinction, plus nuancée, est suggérée par A. Reformatskiji ${ }^{24}$ : la terminologie est principalement liée au système conceptuel de la science dont elle étudie les termes, tandis que la nomenclature se contente d'un étiquetage de ses objets. La nomenclature se caractérise par son lien étroit avec la chose; c'est une liste de termes correspondant à autant de choses, un système de moyens conventionnels visant à établir des perspectives plus commodes en vue de nommer les objets et les choses. Elle est détachée de la langue usuelle. Quant aux terminologies, elles sont nées à partir d'éléments chargés de signification et ont grandi en étroite liaison avec les mots de la langue commune. Si on les analyse dans leur totalité, composées à la fois de substantifs, de verbes et d'adjectifs, on y

22. Vocabulaire de la terminologie, Recommandation ISO/R 1087, juin $1969, \mathrm{p}$. 13. Voir aussi à ce propos : E. Wüster, "Die allgemeine Terminologielehre", Actes du $3^{\mathrm{e}}$ Colloque de linguistique appliquée, Copenhague, 1972 (à paraître) ; L. Drozd, "Methodische Fragen der terminologischen Sprachbehandlung $n$, ibid.; Spang-Henssen, Henning, «Terminology-1, Terminology-2 and Terminology-3 $»$, ibid.

23. J. Maillot, par exemple, op. cit., p. 144.

24. A. Reformatskiji, «Chto takoje termin i terminologija », Voprossi terminologii, Moscou, 1961, p. 47. 
retrouve les mêmes accidents que ceux qui atteignent les mots du vocabulaire commun, c'est-à-dire l'incohérence, la récurrence, la polysémie ou la concurrence. Il suffit pour s'en convaincre d'étudier les valeurs de deux adjectifs farouchement rivaux : atomique et nucléaire.

Disons, d'entrée de jeu, qu'ils ont tous deux leurs lettres de noblesse, ne sont-ils pas, l'un et l'autre, de formation savante? Pourtant, bien des choses les séparent. Le mot atome (du grec : alpha - privatif et radical temnein $=$ couper) a originairement, dans toutes les langues modernes, le sens que lui attribuaient les penseurs de la Grèce antique : celui d'élément constitutif de la matière, indivisible et homogène.

Il y a 2400 ans, Leucippe de Milet et Démocrite d'Abdère avaient échafaudé un univers composé de petites particules qu'ils baptisèrent atomos. L'atomiste Démocrite alla même jusqu'à suggérer l'existence d'une unité ultime insécable l'atome universel. Dans cette cosmogonie, les atomes avaient une forme et pouvaient être pointus, ronds, concaves, sinon crochus, en quel cas leur configuration était telle qu'ils pouvaient s'accrocher les uns aux autres pour constituer les corps. Aujourd'hui, les atomes sont marqués, percutés, dépouillés, excités, ionisés, mésoniques, mésiques, muoniques, interstitiels, légers ou chauds! Le signifiant demeure mais recouvre un signifié tout différent. Sans doute, l'identité foncière du concept était-elle assez forte pour que l'étiquette soit conservée. Toujours est-il qu'aujourd'hui l'atome s'est sécularisé, depuis Gassendi semble-t-il, matérialisé et détaché de ses origines grecques. Dans son ouvrage célèbre Die Physik der Atomkerne, Werner Heisenberg écrivait : "Pour les Grecs, la notion d'atome était encore un moyen pour comprendre le monde dans son ensemble et donner ainsi une explication de tout ce qui pouvait être perçu de la réalité. Maintenant ce sera le moyen de comprendre le comportement de la matière brute et sans vie ${ }^{25}$. 》

La valeur étymologique de corpuscule indivisible, éclipsée dans la physique contemporaine, demeure perceptible dans la chimic traditionnelle qui étudie d'ailleurs l'assemblage des atomes en molécules. En physique nucléaire, on a retenu le mot atome pour désigner un ensemble électriquement neutre composé d'un noyau (protons et neutrons) entouré d'électrons. Le relâchement des rapports étymologiques est un phénomène fréquent en sémantique, lequel s'explique par une inertie linguistique qui nous permet de dire «le soleil se lève», ou permet de créer des locutions d'aspect antithétique telles que atome fissile, qui est l'atome susceptible de subir une fission par absorption de neutrons.

Les structures de l'univers ne se reflètent pas automatiquement dans les structures du langage.

L'adjectif nucléaire a, lui, un pedigree plus modeste. Il vient du latin nucleus qui désigne la noix, plus spécialement le noyau du fruit. Le terme noyau a été rattaché au latin populaire nodellus, de nodus : «nœud». C'est en botanique au sens de « relatif au noyau de la cellule» que l'adjectif nucléaire a été utilisé pour la première fois, et vers 1930 , il a pris le sens de « relatif au noyau de l'atome ».

25. W. Heisenberg, Die Physik der Atomkerne, Braunschweig, 1949, p. 6. 
Dans l'usage moderne, atomique a sur son rival nucléaire l'avantage de l'antériorité. On a dit et on dit encore bombe atomique, pile atomique, et non point bombe nucléaire et pile nucléaire. Les deux grandes conférences de Genève, en 1955 et 1958, ayant pour objet l'utilisation pacifique de l'énergie nouvelle, étaient bel et bien des conférences atomiques. Il n'en reste pas moins que l'énergie en question est celle qui est contenue dans les noyaux d'uranium et de thorium et que la discipline qui en étudie les secrets est la physique nucléaire. Ce n'est pas la physique atomique. Dès l'instant où les physiciens ont pu préciser la microarchitecture de la matière (les noyaux, atomes et molécules) et qu'il s'est trouvé que les réactions étudiées par la chimie traditionnelle avaient lieu entre des molécules, entre des ions et assez rarement entre les atomes, l'existence des adjectifs nucléaire et atomique a entraîné une redistribution au sein du lexique. Les réactions entre noyaux ne pouvaient s'appeler que réactions nucléaires et l'adjectif atomique. est resté disponible, pas bien longtemps pourtant. Aujourd'hui, on possède un système lexical à trois termes interdépendants et se définissant chacun par opposition aux autres. Il est vrai que les distinctions restent peu précises ${ }^{26}$.

L'analyse dite componentielle fait apparaître trois sèmes distincts pour chacun des deux adjectifs et une distribution particulière des rapports sémiques. Nucléaire porte le sème fondamental : relatif au noyau et, dans ce cas il commute avec : «du noyau»; ou encore avec : «du nucléon». Exemples : potentiel nucléaire = énergie potentielle d'un nucléon ( 1 constituant $=$ proton ou neutron), chimie nucléaire $=$ étude des transmutations des nucléides et noyaux, fusion nucléaire $=$ fusion du noyau $;$ instabilité nucléaire $=$ instabilité du noyau, niveau nucléaire $=$ énergie à laquelle un noyau peut exister.

En revanche, le sème fondamental de atomique est : relatif aux atomes. Exemple : poids atomique.

Le deuxième sème spécifique est : relatif à l'énergie libérée par fission ou fusion, nucléaire commute alors avec atomique, ce dernier ne correspondant pas à la réalité physique puisque seul le noyau libère l'énergie. Exemple : énergie nucléaire = libérée par réaction de fission-fusion (= syn. énergie atomique $)$.

Nous pouvons relever un troisième trait sémique : nucléaire qui utilise ou produit l'énergie issue de la désintégration des noyaux d'atomes. Exemple : réacteur nucléaire ou réacteur atomique.

L'adjectif porte un quatrième sème : «guerre avec l'arme nucléaire », dont nous retrouvons la présence dans : guerre nucléaire, chantage nucléaire, conflit nucléaire, force de frappe nucléaire, pat nucléaire.

D'une brève analyse de la terminologie relevée dans les journaux et périodiques non techniques, il ressort assez nettement que l'adjectif nucléaire est en expansion dans les textes ayant trait à l'armement, aux engins ou aux techniques

26. Dans les milieux de spécialistes, on fait aujourd'hui la distinction entre la physique atomique, la physique nucléaire, et la physique corpusculaire. La physique atomique est l'étude des électrons de l'atome c'est-à-dire de sa partie périphérique, le domaine de la physique nucléaire est le noyau de l'atome, ses propriétés et leurs nombreuses applications. L'étude des particules élémentaires fait l'objet d'une science que l'on appelle la physique corpusculaire. 
stratégiques. Sur 70 occurrences, on retrouve 11 fois atomique pour 57 fois nucléaire, soit environ $80 \%$ des fois. «Armes » ou «armements 》 appellent à eux, dans $90 \%$ des cas, l'adjectif nucléaire, alors que le substantif énergie n'a pas de préférence pour l'un ou l'autre adjectif, $50 \%$ pour chacun d'eux. En groupant les fréquences, on constate que le mot atomique n'a cessé de perdre du terrain dans le domaine non scientifique. Par contre, l'adjectif nucléaire a pris des forces. Les fréquences inversement proportionnelles de ces deux adjectifs en fonction de la nature des textes, permettent de constater dans le vocabulaire spécial et dans le vocabulaire commun des renversements sémantiques intéressants.

Les néologismes syntagmatiques, du type énergie nucléaire, bombe atomique, formés d'un substantif et d'un adjectif de relation abondent par suite de la facilité du transfert sémantique d'un domaine d'activité à un autre par le biais de l'adjectif ${ }^{27}$. Les adjectifs de relation constituent un groupe encore mal étudié. Dans les composés du type énergie nucléaire, l'adjectif ne peut en aucun cas se placer devant le substantif, n'a ni degrés de comparaison, ni degrés d'intensité. Pour Bally, que nous suivons ici, «l'adjectif dit de relation transpose des substantifs sans rien changer à leur valeur de substantifs ». Dans : chimie nucléaire, fusion nucléaire, instabilité nucléaire, caractéristique nucléaire, niveau nucléaire, l'adjectif transpose le substantif noyau. Si l'on accepte ces compositions, il en va tout autrement de certains syntagmes épithétiques qui sont frappés d'interdit par les grammairiens. L'adjectif nucléaire se rencontre encore dans les expressions force nucléaire et potentiel nucléaire, et là, le sens change en fonction de la nature du texte. Pour l'atomicien, le potentiel nucléaire est l'énergie potentielle d'un nucléon en fonction de sa position, pour l'homme politique il s'agit de la puissance d'un pays en armes atomiques. La force nucléaire est, pour le premier, toute force autre qu'électrostatique ou électromagnétique s'exerçant entre les nucléons, pour le second, il désigne la force de frappe d'un pays doté d'armes atomiques.

Les relations qui existent entre les éléments des syntagmes propulsion nucléaire, droit nucléaire, accident nucléaire, puissance nucléaire ou assurance nucléaire ne sont pas précisées et c'est le contexte qui est chargé d'expliciter l'acception particulière à retenir. Aucun critère formel ne permet de les distinguer. Les expressions par lesquelles on pourrait remplacer ces syntagmes sont extrêmement encombrantes, car pour assurance nucléaire, il faudrait dire "régime d'assurances régissant les centrales utilisant l'énergie nucléaire ». Tout bien considéré, personne ne se méprendra sur ces tournures elliptiques. L'important est, toutefois, de ne pas outrepasser. Travailleur radiologique (D. Strahlenarbeiter) pour travailleur dans le secteur radiologique, accident radiologique, hygiène nucléaire (D. Strahlenschutz), maladies nucléaires, se situent probablement à la limite et laissent hésitant. Les documents scientifiques sont atteints aujourd'hui - il suffit d'ouvrir les yeux - d'une adjectivité aiguë qui s'explique par le déferlement anglo-saxon, mais aussi parce que la construction avec l'adjectif a une acception plus générique.

Troisième hypothèse : «dans la mesure où les terminologies sont structurées, leur structuration ne correspond pas aux normes du langage, mais aux points de

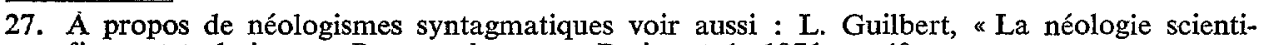
fique et technique», Banque des mots, Paris, $\mathrm{n}^{\circ} 1$, 1971, p. 49. 
vue et aux exigences des sciences et techniques, concernant la réalité même des choses»(p. 16).

Nous admettrons avec E. Coseriu que les 300000 termes de la nomenclature chimique sont rigoureusement structurés du point de vue de la chimie et qu'il serait vain de vouloir établir la «structure sémantique» de ce vocabulaire sur la base de sa structuration technique. En décrivant ainsi l'organisation de ce vocabulaire, le linguiste décrivait non point la configuration du stock lexical lui-même, mais celle des objets signifiés auxquels les différents lexèmes renvoient. L'identification de structures lexicales se ramènerait, dans ce cas, à expliciter une configuration déterminée par la réalité objective, par exemple la tendance de structurer le vocabulaire de l'acier en prenant appui sur les classifications précises des produits et leurs désignations contraignantes. Ces classifications existent et relèvent de la réalité extra-linguiste qui range les aciers d'après leur degré de finition (stade de leur fabrication), leurs formes et dimensions et enfin leur aspect. Pour le métallurgiste, l'ensemble des produits sidérurgiques est groupé en trois catégories : produits bruts, demi-produits et produits finis. Le but du linguiste est, au contraire, d'expliciter par l'analyse ce qui est implicitement structuré dans le répertoire lexical. Ce qui frappe, si l'on analyse l'ensemble du lexique, c'est le grand nombre de termes qui désignent, représentent et classent effectivement les objets mais surtout le fait que les délimitations des termes scientifiques et techniques sont, plus souvent que ne le semble suggérer E. Coseriu, des délimitations dans l' «intuition de la réalité » comme les structurations linguistiques elles-mêmes. De plus, et $M$. Larochette l'a déjà souligné dans son commentaire ${ }^{28}$, un terme scientifique ne désigne pas une réalité de monde extérieur qu'il suffit de reconnaître; en physique nucléaire ou en physique des quantas, le terme ne désigne d'ailleurs plus l'« objet» extérieur, car l'homme de science ne peut le reconnaître ${ }^{29}$. Le terme scientifique désigne une réalité pensée, transposée par l'homme ; son sens correspond à l'idée née de la connaissance d'une chose. L'homme projette dans cette réalité sa vue de celle-ci et doit la désigner par un terme, créant ainsi un « objet » qui n'est pas nécessairement une donnée immédiate. Au sein du domaine confus de l'environnement non linguistique, la langue opère au moyen de signes des découpages, différents d'une langue à l'autre. Voici quelques exemples de découpages :

Ex. 1 : la durée de mise en température ou la durée de chauffage est le temps écoulé entre le début du chauffage et le moment où l'on estime que la température recherchée est atteinte, à cour ou dans la partie de la pièce qui doit être traitée (D. Erwärmdauer). Cette opération considérée en bloc par le français est découpée par l'allemand en un binôme : 1) Anwärmdauer (durée au bout de laquelle la température visée est atteinte en surface, $\left(F_{0}=\right.$ durée de chauffage $)$; 2) Durchwärmdauer : laps de temps compris entre le moment où la température est atteinte par la surface et celui où elle est atteinte par le cœur.

28. Larochette, dans E. Coseriu, "Structure lexicale... », p. 70-71 (interventions préparées sur le rapport de E. Coseriu)

29. H. Pausch, « Anmerkungen zu Problemen und Strukturen der Wissenschafts-sprache in der modernen Physik $»$, Wirkendes Wort, $\mathrm{n}^{\circ}$ 6, novembre 1971, p. 411-424. 
Ex. 2 : la surchauffe est un chauffage effectué dans des conditions de température et de durée telles qu'il se produit un grossissement exagéré du grain. L'allemand fait la distinction entre Überhitzen si la surchauffe résulte d'une température trop élevée et Überzeiten si le chauffage est d'une durée trop longue.

Ex. 3 : On appelle déformation de trempe toute variation des dimensions ou de la forme d'un produit consécutive à un traitement thermique. L'allemand parlera de Verziehen s'il veut évoquer la modification de forme ou de dimensions et Massänderung s'il veut évoquer la modification de dimensions sans changement de forme.

Ex. 4 : la décarburation est la réduction de la teneur en carbone sous l'action du milieu extérieur (appelée en D. Entkohlung). L'allemand distingue la Auskohlung qui est la décarburation totale ou quasi totale et la Abkohlung = décarburation partielle.

Ces exemples prouvent que l'homme découpe la masse de l'expérience d'après le critère des unités lexicales et que ces découpages ne sont pas superposables d'une langue à l'autre. Il en résulte que la structure sémantique n'est pas le simple décalque de la réalité extra-linguistique.

Il faudrait revoir la toute-puissance accordée à l'objet qui prime, domine, sinon opprime, dans la langue spéciale. L'analyse des exemples montre que la langue spéciale structure l'univers à sa manière ou du moins conditionne une analyse du monde extérieur qui lui est propre. Les termes techniques ont une valeur qui se définit par leurs oppositions et par leur fonctionnement, et non point par des critères réels inscrits dans les limites, précises ou imprécises, existant dans lés phénomènes de la réalité. On a jusqu'ici fait ressortir trop nettement la différence entre une réalité objective et tangible dont la langue spéciale n'est que le reflet et une réalité plus subjective, mouvante, vivante, décrite par le langage.

Cette attitude s'explique. Les postulats néo-humboldtiens semblent être en contradiction avec l'existence d'une réalité constante, tangible et antérieure à la connaissance. Pourtant, le savant qui décrit un phénomène, ne décrit jamais qu'un élément arbitrairement isolé de l'univers et procède lui aussi à des découpages que la nature ignore et qui sont forcément sa création. Il segmente des continuums. Lorsque l'on compare la terminologie d'un même domaine en plusieurs langues, on constate la complexité des points de vue et la multiplicité des «visions » d'un même phénomène, chaque observation «engendrant» presque le phénomène.

Quatrième hypothèse : «Toutefois, la connaissance des choses et les idées et opinions à propos des choses ne sont pas sans intervenir dans le fonctionnement du lexique [...] Tout d'abord, la connaissance des choses intervient dans l'interprétation des mots composés et dérivés dont la fonction désignative pourrait être ambiguë 》 (p. 22-23).

Les terminologies scientifiques ont toujours eu largement recours aux procédés de dérivation, de suffixation, par essence même synthétiques, et de composition syntagmatique. Les connexions diffèrent des unités lexicales simples en ce sens que la forme choisie est déterminée ici par un modèle déjà existant dans la 
langue. Le recours à ce modèle présuppose une analogie, laquelle postule à son tour un système de dérivation ou de composition permettant de sauvegarder l'établissement d'un rapport de signification entre les dérivés et la base. Les lexies préfixées et suffixées occupent donc une place intermédiaire entre la juxtaposition pure et simple et la composition.

La structure lexicale des terminologies nouvelles laisse apercevoir, à côté des compositions syntagmatiques, des suffixations et préfixations dont l'ensemble forme, semble-t-il, moins de $50 \%$ du lexique. Étroitement liée au vocabulaire de la chimie et de la physique, la terminologie nucléaire ${ }^{30}$ montre elle aussi une prolifération de suffixes d'origines grecque et latine, ce sont les suffixes : -ide, -ique, -topique, -stat, -ergie, -ion, -oïde, les préfixes photo-, radio-, trans-, thermo-, télé-. Ceux-ci sont souvent à double entente, par exemple : aéro-, télé-, auto-, radio- ${ }^{31}$. Il est apparu qu'une motivation morphologique n'entraîne pas nécessairement une motivation sémantique quoique les analyses structurales conduites sur la dérivation et la composition se soient jusqu'ici révélées les plus fructueuses.

Les terminologies nouvelles usent très volontiers de la composition syntagmatique qui consiste en une collocation de plusieurs lexèmes joints ou non par un ligament. Elle est la formation de base des textes scientifiques, appelée à une production infinie. À ce titre, elle intéresse le lexicographe et plus encore l'auteur de dictionnaires bilingues. De plus, elle ne correspond pas d'une langue à l'autre. L'allemand use de la prédétermination pour intégrer à la base nominale ou verbale un trait pertinent du référent, là où le français use d'adjectifs de relation ou de postdétermination. En prenant pour critère de classement la présence ou l'absence de joncteurs, on peut distinguer : a) la composition syntagmatique asyndétique ou elliptique du type stylo dosimètre; b) la composition syntagmatique épithétique, du type droit nucléaire ; c) la composition syntagmatique avec joncteur : c'est la synapsie de Benveniste ${ }^{32}$, du type tube de comptage.

Les deux derniers types de composition sont parfois en concurrence. On assiste au passage d'un énoncé de dimensions assez vastes comme le syntagme phrastique à articulations complexes, à un énoncé synthétique, syntagme ou lexie préfixée.

La compétition entre les synapsies (dose de rayonnement) et les syntagmes épithétiques correspondants (dose radiologique) n'est pas moins ardente que celle qui oppose, dans d'autres cas, les syntagmes épithétiques (protection radiologique, radiographie neutronique) aux néologismes morphologiques (radioprotection, neutrongraphie). Par ailleurs, polymérisation par rayonnement commute avec radiopolymérisation, sensibilité aux rayonnements avec radiosensibilité et mal des rayons (maladie radiologique) avec radiotoxémie. Ainsi, le préfixe radioentame-t-il une carrière nouvelle avec radio-exposition, radio-élément, radioactivimètre (le trait d'union n'est là que pour éviter une collision de deux voyelles), radiobiologie, radiocarottage, radiométallographie, radioprotecteur, radiorésistance.

30. R. Goffin, « Réflexions sur la terminologie... », p. 25.

31. J. Peytard, "De l'ambiguité sémantique dans les lexies préfixées par auto- », Langue française : la sémantique, $\mathrm{n}^{\circ} 4,1969, \mathrm{p} .88-107$.

32. E. Benveniste, "Formes nouvelles de la composition nominale », Bulletin de la Société française de linguistique, 1966, fasc. $1, \mathrm{n}^{\circ} 61, \mathrm{p} .82-95$. 
Dans la synapsie, nécessairement analytique, les membres sont reliés par des joncteurs ou ligaments synaptiques : $70 \%$ des synapsies présentent le joncteur de, $20 \%$ les joncteurs $\grave{a}$ ou par, sous ou parfois un participe, alors que dans la composition savante la joncture est caractérisée par la finale - $o$ - empruntée des modèles grecs.

Dans le cas des réacteurs nucléaires, la caractéristique distinctive, indiquée par le ligament synaptique $a$, peut porter sur les paramètres suivants : $a$ ) le système de refroidissement primaire : réacteur à circulation forcée (E. forcedcirculation reactor) $; b$ ) la nature de réfrigérant : réacteur à eau légère sous pression (E. pressurized light water reactor) ; c) la géométrie du réacteur ou la structure du cour : réacteur à tubes de force (E. pressure tube reactor), réacteur à cour dilué (E. diluted reactor) ; $d$ ) la nature du combustible : réacteur à combustible en suspension (E. slurry reactor) ; e) la nature du modérateur : réacteur à graphite (E. graphite reactor).

On peut en déduire que l'étude des synapsies ne peut se faire par la seule méthode structuraliste et qu'il faut faire appel à la connaissance de la réalité pour comprendre la nature du désigné. L'espoir exprimé par Benveniste, selon lequel la synapsie en tant que mode de désignation tend à réaliser la limitation de l'arbitraire, reste souvent vain.

On peut se demander pour conclure quels sont les critères auxquels répond la structuration lexicale des terminologies. Sont-ils de nature extralinguistique, auquel cas ils se trouveraient prédéterminés dans les choses signifiées et auraient une motivation objective ou sont-ils, au contraire, de nature linguistique, auquel cas ils seraient inhérents au stock lexical lui-même et auraient une motivation plus subjective ${ }^{33}$. En fait, il est apparu tout au long de nos réflexions qu'il y a tout à la fois parallélisme et interaction entre deux plans; d'une part, le plan de la réalité extralinguistique ou la situation : l'évolution des sciences entraîne l'homme de science à préciser, à diversifier son expérience des choses et à établir de nouveaux classements taxinomiques qui trouvent leur reflet dans le langage; d'autre part, le plan de la réalité linguistique où s'organise «linguistiquement " cette connaissance et où se déclenchent des phénomènes qui viennent transformer l'équilibre instable des relations lexicales, soit par le jeu de la concurrence entre paradigmes (néologismes, emprunts aux vocabulaires voisins) et syntagmes (épithétiques, asyndétiques ou synaptiques), soit entre les lexèmes néologiques, les syntagmes définissants et les ensembles phrastiques ${ }^{34}$.

Une partie se joue donc parallèlement à la réalité extralinguistique et qui répond à des mécanismes linguistiques. La structuration lexicale ne dépend pas seulement $\mathrm{du}$ jeu aléatoire du progrès scientifique mais résulte d'un équilibre entre deux contraintes : celle du monde extérieur et celle du système immanent de la langue.

33. E. Coseriu, "Structure lexicale...", p. 22 : "le langage classe la réalité, mais il le fait selon des intérêts et des attitudes humaines $»$.

34. J. Dubois, « Les problèmes du vocabulaire... ", p. 196. 
Les auteurs de dictionnaires ou de glossaires bilingues ou multilingues ${ }^{\mathbf{3 5}}$ sont fréquemment partis du principe de l'existence de structures objectives au sein des terminologies. Nous avons cru pouvoir montrer d'une part que les découpages de la réalité extralinguistique pratiqués par les langues fonctionnelles ne sont presque jamais superposables d'une langue à l'autre et d'autre part que l'on retrouve dans les terminologies les mêmes accidents que ceux qui frappent les mots du vocabulaire commun (incohérence, polysémie). Il en résulte que la lexicographie multilingue se doit de tenir compte du fait que la langue fonctionnelle n'est pas une nomenclature ou un simple inventaire de mots dans lequel il suffirait de changer l'apparence du signifiant (l'étiquette) pour passer d'une langue de départ à une langue d'arrivée. La réalité demeure fragmentée et liée à la diachronie de l'expérience que l'homme acquiert du monde par le langage. L'auteur du dictionnaire multilingue fera bien de ne plus présenter les mots dans leur isolement théorique, les unités lexicales ne se correspondant pas d'une façon biunivoque et les termes n'occupant pas une position identique dans la mosaïque des champs lexicaux. Cette constatation vaut aussi, nous l'avons dit, pour les terminologies scientifiques. La hiérarchie des structures lexicales y varie, elle aussi, d'une manière sensible d'une langue à l'autre. Les déficiences relevées dans les dictionnaires bilingues (ou multilingues) scientifiques de conception traditionnelle tiennent donc surtout à l'absence de données «sémantiques », telles que l'extension ou la délimitation des aires sémantiques. Le lexicographe devra faire ressortir les différences lorsqu'il y a «choix 》, et, toutes les fois où le même mot fonctionne tantôt comme générique tantôt comme spécifique. Il fera normalement appel aux données du micro-contexte. L'information prendra la forme d'une définition ou de termes situés dans leur environnement contextuel. Les microcontextes des glossaires peuvent être sélectionnés tout à la fois suivant le critère linguistique et le critère informatif. Le glossaire contextuel multilingue ${ }^{3 e}$ consacré à une terminologie s'apparente au dictionnaire de choses mais il est aussi, et peut être avant tout, un dictionnaire de langue.

Le corpus comporte dans ce cas les éléments suivants : a) des définitions de termes, tirées de textes originaux ou normalisés, mis en parallèle; la mise en parallèle permet de « découvrir» certaines réalités; $b$ ) des exemples d'emploi des mots dans un contexte pertinent aux plans terminologique et informatif, le contexte permettant d'établir le statut sémantique du terme ; $c$ ) à titre exceptionnel, de simples équivalences de termes, si les correspondances ont un caractère biunivoque.

35. A ce propos, nous avons consulté les ouvrages ou articles suivants : J. Dubois et C. Dubois, Introduction à la lexicographie. Le dictionnaire, Paris, Larousse, 1971; L. Guilbert, «Dictionnaire et linguistique : essai de typologie des dictionnaires monolingues français contemporains ", Langue française : le lexique, 1969, p. 8ss.; K. Baldinger, "Semasiologie und Onomasiologie im zweisprachigen Wörterbuch", Interlinguistica, Mélanges Wandruszka, p. 384-396.

36. Quelques exemples de glossaires contextuels : Glossaire des normes de l'acier, (4 langues), publié par le Bureau de terminologie de la Commission des Communautés européennes, Bruxelles, 1972; Glossaire phraséologique (5 langues), l'Acier dans les industries chimiques, publié par le Bureau de terminologie de la Commission des Communautés européennes, Luxembourg, 1968. 
Il ressort de ces réflexions que le lexicologue structuraliste pourrait tirer parti de l'étude des terminologies scientifiques qui constituent des ensembles lexicaux aux structures sémantiques à la fois originales et proches du reste du lexique, en tout cas privilégiées. S'appuyant sur les résultats d'une réflexion épistémologique alliée à une systématisation croissante, la lexicologie multilingue et la lexicographie jugées trop longtemps comme des disciplines indignes d'être admises dans le sanctuaire des sciences de l'esprit, pourront ainsi entrer de plain-pied dans la phase scientifique et même de façon exemplaire.

-

ROGER GOFFIN

\section{QUESTIONS}

$M$. Baldinger : Je suis entièrement d'accord avec la critique que vous avez faite de la théorie de Coseriu quant à la nomenclature scientifique. Coseriu avait présenté cette thèse pour la première fois au $1^{\text {er }}$ congrès de linguistique appliquée à Nancy en 1963 et tous ceux qui sont intervenus dans la discussion ont pris position contre cette conception. Ce serait une espèce d'idéal d'avoir une nomenclature, une terminologie scientifique absolument monosémique qui ne prête plus à équivoque. Au même colloque de 1963, quelqu'un a fait une communication sur les quinze significations du mot "signification", ce qui est déjà une démonstration contre cette thèse de Coseriu. Il y a une seule différence entre le langage général et la terminologie scientifique; dans le langage scientifique j'ai besoin de définir ma terminologie. Je crois que vous avez parfaitement raison de traiter la terminologie comme faisant partie à la fois de la terminologie normale, mais avec un statut spécial à l'intérieur du langage général.

Réponse : Je voudrais souligner ceci, ce n'est pas une réponse directe : si l'article a été présenté pour la première fois en 1963, il a été reproduit plusieurs fois dans différentes revues, et il a donné lieu à des thèses comme celle de Geckeler. Malgré les interventions au congrès de 1963, ces idées ont quand même été propagées, et aujourd'hui encore je constate que, même dans la thèse de Geckeler, ce sont les mêmes idées qui sont reproduites, tronquées là où ça ne marche pas très bien.

M. Rey : Je voudrais quand même faire réfléchir sur une certaine ambiguïté dans les positions de Coseriu et votre critique. Lorsque Coseriu oppose la structuration proprement interlinguistique à une structuration des cho- ses, il ne faut pas prendre le mot "chose" ou le mot " objet » dans un sens ontologique, au sens métaphysique du terme, mais bien l'entendre comme une articulation qui, lorsqu'il s'agit de science, et vous l'avez souligné vous-même, c'est incontestable, se situe au niveau de la conceptualisation. En effet, les choses n'ont d'intérêt en linguistique qu'en sémantique extensionnellè lorsqu'il s'agit de désignation. Quand je parle de votre tableau noir, de ce lutrin sur lequel vous avez posé vos papiers, il $\mathrm{y}$ a une triple désignation parce que les mots tableau noir, lutrin, papiers, ont été mis dans des phrases qui ont des déterminants. Je suis en sémantique extensionnelle. Si on sort les mots de la phrase et qu'on parle de tableau noir, de lutrin ou de papiers, il est évident que la signification de ces trois lexèmes ne peut pas être considérée comme directement référentielle, mais comme une signification qu'on peut analyser au point de vue sémique et à ce moment-là on plonge dans la structure de la langue. Il y a un cas où on peut utiliser des unités lexicales sorties de leur contexte et où c'est la désignation, la référence qui prime, c'est le nom propre. C'est probablement le seul cas. Autrement, je ne pense pas qu'on puisse jamais dire qu'une nomenclature, une terminologie, le paradigme dans la langue, puisse être en relation directe avec les choses. Je ne crois pas du tout que ce soit ce qu'ait voulu dire Coserin. Lorsqu'il s'agit de désignation scientifique, il s'agit de structures qui sont conceptuelles. Vous avez opposé tout à l'heure " atomique » et «nucléaire ", et on le voit très bien avec ces exemples-là : lorsqu'il s'agit de la langue non scientifique, "atomique » et « nucléaire " peuvent fonctionner comme des synonymes absolus. Si la langue emploie « force nucléaire ", " arme nucléaire », elle emploiera indif- 
féremment au plan du sens *arme atomique », «force atomique». S'il y a une fréquence plus grande de l'un par rapport à l'autre, c'est pour des notions sociolinguistiques, de connotation, de fréquence, etc. Par contre, pour un physicien, il n'y a jamais eu la moindre possibilité de confusion entre le concept " atomique " et le concept * nucléaire ». D'ailleurs l'usage de ces termes est réparti différemment suivant les types de discours tenus, car vous rencontrerez « atomique " en physico-chimie parce qu'il s'oppose à : moléculaire ». Vous rencontrerez « nucléaire * en physique pure parce qu'il s'oppose à "atomique ", en tant que atomique nucléaire + électronique. Pour un physicien il n'y a absolument aucune possibilité de confusion, c'est une chose qui n'a strictement aucun rapport. Il y a un rapport de contiguïté, de métonymie, mais conceptuellement il n'y a aucun rapport. Au point de vue historique, atomique était d'abord un terme qui n'était pas scindé tant qu'on n'a pas eu un modèle de l'atome dans lequel le noyau est opposé à la périphérie, aux électrons. Mais du moment qu'on a un modèle conceptuel, on a une oppo- sition sémantique dans le vocabulaire scientifique entre "atomique » et "nucléaire". Cette opposition sémantique n'est pas linguistique puisqu'elle ne se réalisait pas : "nucléaire » existait dans d'autres emplois et \& atomique » avait la totalité du sens. L'opposition est conceptuelle, je crois que c'est dans ce sens qu'on peut parler, d'une part, d'une structuration interlinguistique et, d'autre part, d'une structuration extra-linguistique.

Réponse : Je suis tout à fait d'accord avec vous, mais je voulais montrer essentiellement d'une part, qu'il y a un danger de faire au départ une distinction trop nette entre le vocabulaire scientifique et terminologique, et d'autre part, l'intérêt de l'étude des terminologies pour l'ensemble des études lexicales. Il faut évidemment raffiner et affiner les distinctions, et ensuite montrer combien les lexicologues traditionnels ont tendance à étudier le substantif, alors que la pratique montre que le terme technique est aussi essentiellement un terme qui décrit des phénomènes, des choses qui bougent, des liquides qui s'écoulent. 